\title{
A Different Content and Scope for School Arithmetic
}

\author{
Patricia Baggett \\ New Mexico State University, USA \\ Andrzej Ehrenfeucht \\ University of Colorado, USA
}

Modern school arithmetic has evolved from methods that were used to teach 19th century accountants. Today its scope is broader, but the sequence of topics remains almost the same. The goal of teaching arithmetic has changed from achieving speed and accuracy in written computation to understanding mathematical principles and the structure of algorithms. Attempts have been made to reach this goal just by changing pedagogy, but students are still required to master skills of written computation. To carry out written computation, students need good penmanship and instant recall of arithmetic "facts", which is challenging to many, especially those who speak different languages at home and at school. We present a different approach to teaching elementary arithmetic based, not on written computations, but on algorithms executed on counting boards designed on principles invented by John Napier. This approach suggests a radically different sequence of topics and some changes in content, and teaches arithmetic as a tool for solving practical problems in low- and high-tech environments.

Keywords: Arithmetical algorithms, counting boards, scope and sequence.

The history of mathematical notation shows that writing was rarely used as a tool for computation e.g., Chrisomalis (2010), but that computation was more often done with the help of low-tech devices (counting boards). During the last 50 years, modern computing devices have rendered paper and pencil calculations obsolete, but students in early grades need a large amount of hands-on experience to learn the concepts of arithmetic. Written computation is still the main teaching tool for learning those concepts. A variety of other representations of numbers attempt to make arithmetic concepts more concrete, and among them the use of a number line is most often recommended by the Common Core Mathematics Standards (National Governors Association et al., 2009).

John Napier (1617; 1990) in his Rabdology, designed a counting board where values of locations in each column (called a rod) form a geometric progression with factor 2 , and he showed that most arithmetic computations that were known during his time (including computations of roots) could be carried out on his board with the help of just one simple set of regrouping rules. This work was completely ignored, probably because he used only 

integer or its reciprocal, and e is an integer (that can be positive, negative, or zero).

The length $\mathrm{n}$ of a rod is not theoretically important, because any rod may be extended up and down whenever it is needed. In practice, so far, we have never needed to use rods that have more than 10 locations, but we can imagine rods of any length.

Any board has to contain a rod with root 1 , but how other rods are aligned may vary, so the same set of rods can form different boards.

Numbers are represented by configurations of tokens on a board. Each token has its absolute value $\mathrm{v}$, and a local value on a board that is the product of $v$ and the value of the location it occupies. The value of a board is the sum of all local values of the tokens.

It is sufficient to have tokens with only two values, 1 and -1 . We use white tokens to represent 1 and red tokens to represent -1 , as shown in Figure 2.

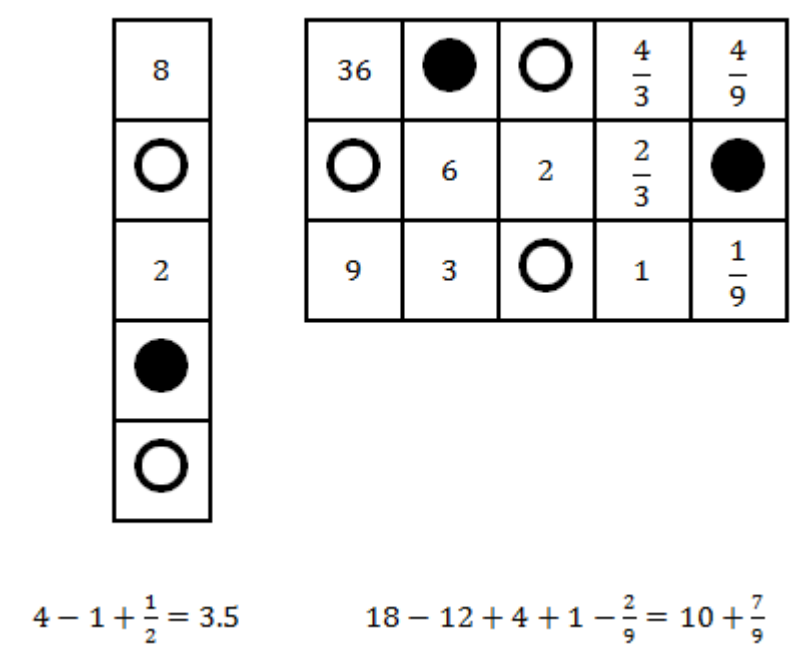

Figure 2. Representing numbers on a board (A white token has the value 1 and a red token, shown in black, has the value -1).

\section{Numbers Represented on One Rod}

(i) When $r=1$, then the numbers that are represented on one rod are integers and also fractions that have powers of two as denominators (binary fractions). Technically it is a small ring of integers in which only the prime number 2 has a reciprocal.

(ii) When $r$ is an odd positive integer, then integers represented on the rod are limited to those that are divisible by r. So they form a subset of the numbers described in (i).

(iii) When $r$ is a reciprocal of an odd positive integer $d>1\left(r=d^{-1}\right)$, then the numbers that are represented consist of all improper fractions with 

An original board

\begin{tabular}{|c|c|c|}
\hline 1 & $\frac{1}{3}$ & $\frac{1}{5}$ \\
\hline$\frac{1}{2}$ & $\frac{1}{6}$ & $\frac{1}{10}$ \\
\hline$\frac{1}{4}$ & $\frac{1}{12}$ & $\frac{1}{20}$ \\
\hline
\end{tabular}

and its extension

\begin{tabular}{|c|c|c|c|c|c|c|}
\hline 30 & 10 & 6 & 2 & $\frac{2}{3}$ & $\frac{2}{5}$ & $\frac{2}{15}$ \\
\hline 15 & 5 & 3 & 1 & $\frac{1}{3}$ & $\frac{1}{5}$ & $\frac{1}{15}$ \\
\hline 7.5 & 2.5 & 1.5 & $\frac{1}{2}$ & $\frac{1}{6}$ & $\frac{1}{10}$ & $\frac{1}{30}$ \\
\hline 3.25 & 1.25 & .75 & $\frac{1}{4}$ & $\frac{1}{12}$ & $\frac{1}{20}$ & $\frac{1}{60}$ \\
\hline
\end{tabular}

Figure 4. A typical case of appending rods to a board.

There is a reason for making this restriction. As we will see below, for each board we have a set of rules for changing the patterns of tokens, which we call "regrouping rules". As it happens, we need exactly one rule for each reciprocal of a prime number. But finding these rules requires factoring a number whose reciprocal is a root. But this is a difficult task for most numbers. So we allow only the extensions that can be handled by rules that are already present.

\section{Regrouping}

Regrouping is changing the configuration of tokens on a board that preserves the sum of its local value. Now we describe the complete set of rules for regrouping. We call the set of rules complete if they enable changing a configuration of tokens into any other configuration that has the same value.

As shown in Figure 5, there are two rules of regrouping tokens on one rod:

(1) A token can be exchanged with two tokens that have the same value on the location just below.

(2) Two tokens of opposite values (1 and -1) can be put on, or removed from, any locations.
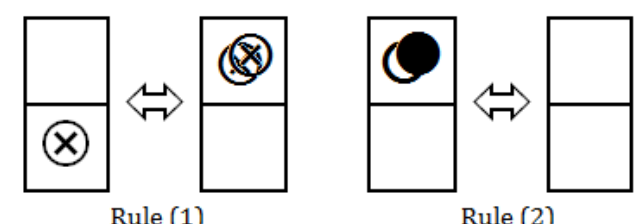

Figure 5. Regrouping tokens ( $X$ in a circle indicates a token that is either white or red).

We define a one-to-one matching between locations of two rods by matching their roots and extending this matching up and down. (This may require adding more locations to a rod as described above.) We say that 

Tokens are two-sided, with colors white and red. White has the value 1 , and red the value -1 . In general, one can put more than one token in each location. But the capacity of an integer board is the biggest integer that is represented with at most one token per location. So the capacities of the integer boards shown in Figure 7 are 12, 42, and 31. Most practical computations on a board are limited to numbers that are at most the double of the board's capacity.
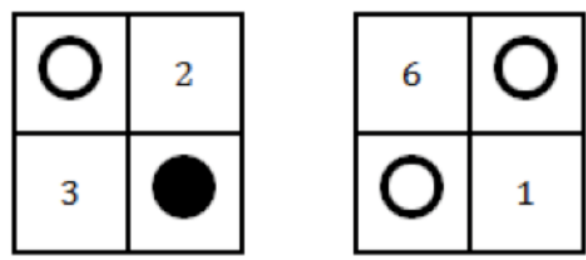

Figure 8. The number 5 is on both boards.

\section{Using Negative Numbers}

Positive and negative integers and fractions can be introduced at the same time because they are governed by the same set of rules. Using fractions is optional, because you may use just boards that have whole numbers as roots, and never use any locations below the root of a rod.

On the other hand, we think that red tokens need to be used, even when negative numbers are not discussed at the same time, as shown on the left in Figure 8. The algorithm for "taking away" positive numbers is more difficult, and harder to use, than the algorithm for adding negative numbers in which "white and red tokens cancel each other". follows:

One can introduce red tokens without referring to negative numbers as

Putting a red token on a board is a request to take out the white token from that particular location. During regrouping, when a white token lands on a red token, they are both taken away and the request is fulfilled.

A similar approach was used in China during the time when computation was done with rods. Red rods were used to represent positive numbers, and black rods were used for subtraction, without being treated as different numbers, but only as numbers to be subtracted (Hart, 2011).

\section{Using Boards in Middle Grades $\left(3^{\text {rd }}\right.$ through $8^{\text {th }}$ )}

Three main topics of elementary arithmetic are covered in middle grades: exact computation with (finite) decimals, exact computation with common fractions, and approximate computation with (finite) decimals. We do not treat the arithmetic of negative numbers as a separate topic, because we have already stated that negative numbers could be used as a computational tool, even before they are introduced as a new mathematical concept. 



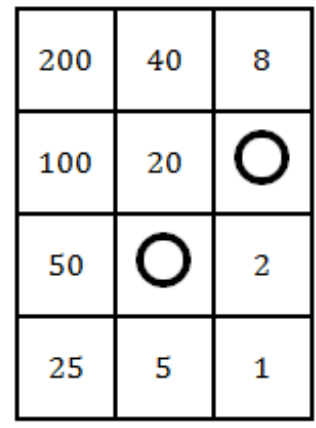

$10+4=14$
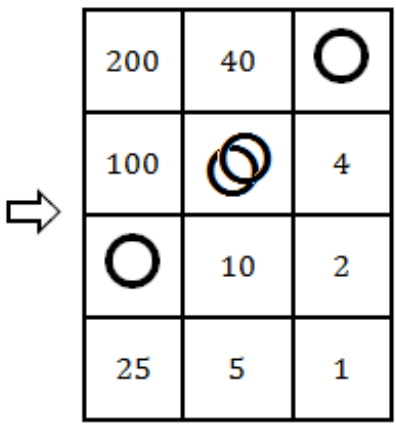

$50+20+20+8=98$

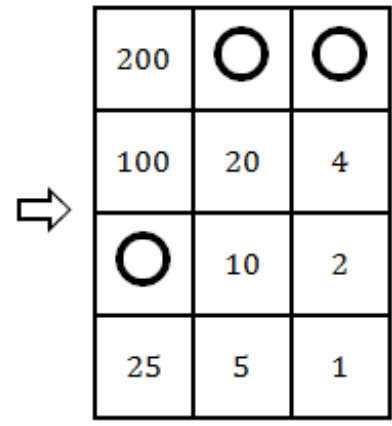

$50+40+8=98$

Figure 10. Multiplying 14 by 7 on a base 5 board.

\section{Comment}

We see that sliding the multiplier along the rod of the multiplicand, and adding its copies as you go along, carries out multiplication. It is essential that values of locations on a rod form a geometric progression. Geometric progressions were essential in the design of logarithms. And sliding one rod along the other was the principle underlying the invention of the slide rule.

\section{Approximate Computation and the Simplest Representations of Numbers}

Numbers that are represented on decimal boards are finite decimals only. They are the smallest set of rational numbers closed under addition and multiplication, which contains all integers and exactly two reciprocals of the prime numbers $2^{-1}=.5$ and $5^{-1}=.2$. This is really a "very small" subset of the rational numbers, so how the other numbers are approximated deserves special attention.

In school mathematics decimals include finite decimals and also infinite periodic decimals, which describe all rational numbers. And in secondary grades all irrational numbers are introduced as non-periodic decimals. So the question of approximations is usually reduced to one rule of "rounding".

\section{Non-Adjacent Forms (NAF)}

NAF notation uses three digits, 0,1 and -1 , to write binary numbers, but it requires that two consecutive non-zero digits are separated by at least one zero (Wikipedia reference).

Every number written in base two has a unique NAF representation, so when we use both red and white tokens on the same rod, every number has a unique representation, with the restriction that no two adjacent locations are occupied. Non-adjacent forms also use the smallest possible number of tokens that are needed to represent a number on any given rod. This representation has many uses and is more accurate when numbers are rounded or truncated, and it can 



\section{Teaching Common Fractions Using Counting Boards}

We give here an example of how to use counting boards to teach common fractions by using a specific board that handles fractions with denominators that are factors of 60 , namely, 2, 3, 4, 5, 6, 10, 12, 15, 20, 30, and 60 . The operations that are allowed are addition and subtraction only. This method is still feasible when we take any number smaller than 1000 (instead of 60), but it starts being too complex for bigger numbers with many factors.

\section{Addition and Subtraction of Fractions whose Denominators are Factors of 60}

\begin{tabular}{|l|l|l|l|}
\hline 60 & 20 & 12 & 4 \\
\hline 30 & 10 & 6 & 2 \\
\hline 15 & 5 & 3 & 1 \\
\hline
\end{tabular}

\begin{tabular}{|c|c|c|c|}
\hline 1 & $\frac{1}{3}$ & $\frac{1}{5}$ & $\frac{1}{15}$ \\
\hline$\frac{1}{2}$ & $\frac{1}{6}$ & $\frac{1}{10}$ & $\frac{1}{30}$ \\
\hline$\frac{1}{4}$ & $\frac{1}{12}$ & $\frac{1}{20}$ & $\frac{1}{60}$ \\
\hline
\end{tabular}

Figure 12. An integer board and its corresponding fraction board with tokens to be added.

On the fraction board we have a number, $1 / 2-1 / 3+1 / 4-1 / 5$, as shown on the right in Figure 13 To compute the total we put the same configuration of tokens on the integer board, as shown in the left in Figure 13.

Figure 13. The same configuration of tokens is put on the integer board. Now we regroup the tokens in Figure 13, getting $30-20+15-12+3=13$, as shown in the left in Figure 14. 


